\title{
Autonomic Regulation of the Cardiovascular System: Diseases, Treatments, and Novel Approaches
}

\author{
Zixi Jack Cheng ${ }^{1}$ (D) $\cdot$ Renjun Wang ${ }^{2} \cdot$ Qing-Hui Chen $^{3}$ (B)
}

Received: 28 December 2018/ Accepted: 3 January 2019/Published online: 19 January 2019

(C) Shanghai Institutes for Biological Sciences, CAS 2019

The autonomic nervous system (ANS) integrates the involuntary physiological activities of visceral organs that are vital for survival. In particular, the ANS controls heart rate, blood pressure, breathing, gastrointestinal contraction and secretion, and electrolyte and fluid homeostasis. Many studies have focused on understanding the neural mechanisms of autonomic dysfunction or neuropathy in pathophysiological states including hypertension, heart failure, orthostatic hypotension, stroke, hypoxia, sleep apnea, diabetes, and metabolic disorders. In these diseases and conditions, the function of the ANS is altered, leading to autonomic neuropathy. The effect of autonomic neuropathy ranges from mild to life-threatening. However, the mechanisms of autonomic neuropathy in the pathological states are not well understood and have yet to be elucidated.

For this special issue, the Editor-in-Chief of Neuroscience Bulletin invited Dr. Qing-Hui Chen as a Guest Editor to organize and gather a number of investigators in this field to contribute original research articles that address the underlying neural mechanisms and significance of autonomic dysfunction in the pathogenesis of these diseases and provide potential interventions. Furthermore, the

Zixi Jack Cheng

Zixi.Cheng@ucf.edu

Qing-Hui Chen

qinghuic@mtu.edu

1 Burnett School of Biomedical Sciences, College of Medicine, University of Central Florida, Orlando, FL 32816, USA

2 Department of Biotechnology, School of Life Science, Jilin Normal University, Siping 136000, China

3 Department of Kinesiology and Integrative Physiology, Michigan Technological University, Houghton, MI 49931-1200, USA invited review articles contribute to the recent understanding of the mechanisms of autonomic function and dysregulation, which may provide novel approaches and strategies to decipher the complicated autonomic neural network.

Hypertension is a devastating cardiovascular disease. In this issue, spontaneous hypertension, salt-sensitive hypertension, and obesity-related hypertension are discussed. Sympathetic overactivity is currently considered as an important mechanism for the development of hypertension. The activation of pre-sympathetic neurons in the hypothalamic paraventricular nucleus (PVN) and the rostral ventrolateral medulla (RVLM), the main source of excitatory synaptic input to sympathetic preganglionic neurons in the spinal cord, both contribute to sympathetic overactivity. $\mathrm{Li}$ and colleagues review the cellular and molecular mechanisms underlying the hyperactivity of pre-sympathetic neurons in the PVN which may lead to the increase in sympathetic outflow in spontaneously hypertensive rats (SHRs), a model of primary hypertension [1]. Kang and colleagues examine the antioxidative effect of Metformin (MET) in salt-induced hypertension. Their study suggests that central MET administration reduces sympathetic outflow and arterial pressure (AP) by attenuating oxidative stress, inhibiting the renin-angiotensin system, and restoring the balance between excitatory and inhibitory neurotransmitters in the PVN [2]. Furthermore, Kang and colleagues also show that the increased AP and sympathetic nerve activity (SNA) can be suppressed by blockade of endogenous angiotensin (Ang-1-7) in salt-induced hypertension through the modulation of proinflammatory cytokines and oxidative stress [3]. Using the obesityrelated hypertensive $(\mathrm{OH})$ rat model, Zhou and colleagues investigate the effects of PVN intermedin (IMD) on the sympathetic activation induced by chronic systemic 
application of lipopolysaccharide (LPS). Their study indicates that IMD in the PVN attenuates the SNA and hypertension, and decreases the ERK activation in the LPSinduced enhancement of SNA in OH rats. The IMD effect is mediated by adrenomedullin receptors [4]. In the RVLM of SHRs, Wang and colleagues describe the effect of angiotensin-converting enzyme 2 (ACE2) overexpression on cholinergic synaptic transmission and suggest that ACE2 overexpression in the RVLM attenuates the enhanced cholinergic synaptic transmission, sympathoexcitation, and AP [5].

Arterial baroreflex impairment is associated with the prognosis and mortality of cardiovascular diseases and diabetes. $\mathrm{Li}$ and colleagues have made a significant contribution to the understanding of cellular and molecular mechanisms underlying arterial baroreceptor remodeling in cardiovascular diseases and diabetes. As they point out, arterial baroreceptors can be considered as a potential therapeutic target to improve the prognosis of patients with cardiovascular diseases and diabetes [6]. Chronic intermittent hypobaric hypoxia (CIHH) has been shown to exert anti-hypertensive effects. Zhang and Wang and colleagues show that $\mathrm{CIHH}$ up-regulates nitric oxide synthase in the nucleus tractus solitarii, thus effectively reducing AP in 2-kidney-1-clip hypertensive animals [7]. It is known that excessive reactive oxygen species (ROS), such as the superoxide radical, are commonly associated with cardiac autonomic dysfunctions, including impairment of baroreflex sensitivity. Li and Qiao and colleagues demonstrate that chronic treatment with fenofibrate plays a crucial role in the neural control of AP by improving baroreflex afferent function, possibly due to peroxisome proliferatoractivated receptor alpha-mediated up-regulation of mitochondrial uncoupling protein 2 expression and reduction of oxidative stress [8]. Though superoxide dismutase 1 (SOD1) overexpression may protect against ROS damage to the ANS, superoxide radical reduction may change normal cardiovascular function. Cheng and colleagues tested the effects of overexpressing human SOD1 and examined the functional changes in central and vagal efferent components in the baroreflex arc. They demonstrate that while SOD1 overexpression likely enhances aortic depressor nerve function and the central mediation of bradycardia, it decreases vagal efferent control of heart rate. Thus, SOD1 overexpression may alter the function of neuronal components in the arc differently. Hence, it is important to test the effect of the antioxidant SOD1 on the neural components before treatment [9]. In recent years, vagal stimulation has been used as an effective therapy in treating cardiovascular diseases with autonomic dysfunction. Zang and colleagues thoroughly review the potential drug targets for modulating vagal nerve activation in cardiovascular diseases [10]. Currently, the NIH has a large program called SPARC (Stimulating Peripheral Activity to Relieve Conditions). This program aims to transform the understanding of nerve-organ interactions and ultimately advance the neuromodulation field toward precise treatment of diseases and conditions (https://commonfund.nih. gov/SPARC).

In addition to hypertension and an impaired baroreflex, this special issue also includes five other types of important autonomic neuropathy in patients and animal models. Orthostatic intolerance is a series of clinical symptoms that develop during prolonged standing in the upright position, including frequent, recurrent, or persistent dizziness, fatigue, and heart palpitations with or without syncope. $\mathrm{Du}$ and colleagues review the possible mechanisms of orthostatic hypercoagulability which may be involved in the pathogenesis of orthostatic intolerance [11]. Visceral pain with irritable bowel syndrome (IBS) is a common disease and the mechanism remains largely unknown. Xu and colleagues' work supports the idea that the adrenergic receptor NE- $\beta 2$ signaling pathway plays an important role in the development of visceral hypersensitivity. This study might shed light on the pathogenesis of visceral hypersensitivity imposed by environmental stress during early and adult life. In addition, inhibitors of $\beta 2$ adrenergic receptors might relieve the abdominal pain with IBS [12]. Inflammatory bowel disease may lead to damage of the enteric nervous system. Wang and team examine the cytoprotective mechanisms of body protection compound 157 (BPC157) in the gastrointestinal tract. They report that BPC157 increases the survival of cultured enteric neurons and the proliferation of cultured enteric glial cells, which may improve the healing of damaged enteric nerves and mucosal structures in inflammatory bowel diseases [13]. Diabetes induces cardiovascular autonomic neuropathy (CAN). Weng and colleagues demonstrate that decreased $\beta$-cell function is associated with CAN in newly-diagnosed type 2 diabetic patients. Importantly, they found that parasympathetic nerves are more commonly and severely impaired than sympathetic nerves at the early stage of type 2 diabetes [14]. Cerebral stroke not only affects sensory and motor function, but also often causes autonomic disturbances in patients due to damage of the central autonomic network. Zhang and colleagues review the clinical and experimental findings as well as management strategies of post-stroke autonomic disturbances. It appeared that ANS modulation has become an emerging therapeutic strategy for stroke management in addition to treatments for sensorimotor dysfunction [15].

Finally, two excellent review papers highlight recent advances in novel tools which may decipher the intricacies of complex autonomic networks. In the past few decades, great efforts have been made to study the structure and functions of the autonomic system. However, our 
understanding of the classification of autonomic neuronal subpopulations still remains limited, and a precise map of their connectivity has not been achieved. One of the major challenges that hinder rapid progress in these areas is the complexity and heterogeneity of autonomic neurons. To facilitate the identification of neuronal subgroups in the ANS, Liu and Xu review the well-established and cuttingedge technologies that are frequently used in peripheral neuronal tracing and profiling, and discuss the operating mechanisms, advantages, and targeted applications [16]. Furthermore, Mukerjee and Lazartigues elaborate on the fundamental concepts and applications of the powerful techniques including optogenetics, genetically-encoded $\mathrm{Ca}^{2+}$ indicators, and designer receptors exclusively activated by designer drugs, which have substantially improved our understanding of brain functions [17].

This special issue includes 17 papers. The novel findings reported here increase the understanding of autonomic regulation and dysfunction of the ANS. Furthermore, it provides novel approaches and strategies to study the ANS and potentially treat autonomic neuropathy. We hope this special issue will inspire scientific exchange and collaboration and stimulate growth in this exciting and everdeveloping field.

Acknowledgments We would like to thank all the authors for their informative contributions and reviewers for their constructive critiques. We are grateful to Drs. Jason R. Carter, Yu-Ming Kang, Yulong Li, and Guang-Yin $\mathrm{Xu}$ for their support and assistance during the organization of the special issue.

\section{References}

1. Zhou JJ, Ma HJ, Shao JY, Pan HL, Li DP. Impaired hypothalamic regulation of sympathetic outflow in primary hypertension. Neurosci Bull 2019, 35: 124-132.

2. Yu XJ, Zhao YN, Hou YK, Li HB, Xia WJ, Gao HL, et al. Chronic intracerebroventricular infusion of metformin inhibits salt-sensitive hypertension via attenuation of oxidative stress and neurohormonal excitation in rat paraventricular nucleus. Neurosci Bull 2019, 35: 57-66.

3. Yu XJ, Miao YW, Li HB, Su Q, Liu KL, Fu LY, et al. Blockade of Endogenous angiotensin-(1-7) in hypothalamic paraventricular nucleus attenuates high salt-induced sympathoexcitation and hypertension. Neurosci Bull 2019, 35: 47-56.

4. Sun J, Ren XS, Kang Y, Dai HB, Ding L, Tong N, et al. Intermedin in paraventricular nucleus attenuates sympathoexcitation and decreases TLR4-mediated sympathetic activation via adrenomedullin receptors in rats with obesity-related hypertension. Neurosci Bull 2019, 35: 34-46.

5. Deng Y, Tan X, Li ML, Wang WZ, Wang YK. Angiotensinconverting enzyme 2 in the rostral ventrolateral medulla regulates cholinergic signaling and cardiovascular and sympathetic responses in hypertensive rats. Neurosci Bull 2019, 35: 67-78.

6. Tu H, Zhang D, Li YL. Cellular and molecular mechanisms underlying arterial baroreceptor remodeling in cardiovascular diseases and diabetes. Neurosci Bull 2019, 35: 98-112.

7. Li N, Guan Y, Tian YM, Ma HJ, Zhang XJ, Zhang Y, et al. Chronic intermittent hypobaric hypoxia ameliorates renal vascular hypertension through up-regulating NOS in nucleus tractus solitarii. Neurosci Bull 2019, 35: 79-90.

8. Guan J, Zhao M, He C, Li X, Li Y, Sun J, et al. Anti-hypertensive action of fenofibrate via UCP2 upregulation mediated by PPAR activation in baroreflex afferent pathway. Neurosci Bull 2019, 35 : $15-24$.

9. Chen J, Gu H, Wurster RD, Cheng Z. Baroreflex control of heart rate in mice overexpressing human SOD1: Functional changes in central and vagal efferent components. Neurosci Bull 2019, 35 : 91-97.

10. Liu L, Zhao M, Yu X, Zang W. Pharmacological modulation of vagal nerve activity in cardiovascular diseases. Neurosci Bull 2019, 35: 156-166.

11. Quan W, Wang Y, Chen S, Du J. Orthostatic intolerance and coagulation abnormalities: An update. Neurosci Bull 2019, 35: 171-177.

12. Du WJ, Hu S, Li X, Zhang PA, Jiang X, Yu SP, et al. Neonatal maternal deprivation followed by adult stress enhances adrenergic signaling to advance visceral hypersensitivity. Neurosci Bull 2019, 35: 4-14.

13. Wang XY, Qu M, Duan R, Shi D, Jin L, Gao J, et al. Cytoprotective mechanism of the novel gastric peptide BPC157 in gastrointestinal tract and cultured enteric neurons and glial cells. Neurosci Bull 2019, 35: 167-170.

14. Yang X, Xu W, Zhu Y, Deng H, Tan Y, Zeng L, et al. Decreased $\beta$-Cell function is associated with cardiovascular autonomic neuropathy in Chinese patients newly diagnosed with type 2 diabetes. Neurosci Bull 2019, 35: 25-33.

15. Mo J, Huang L, Peng J, Ocak U, Zhang J, Zhang JH. Autonomic disturbances in acute cerebrovascular disease. Neurosci Bull 2019, 35: 133-144.

16. Liu DS, Xu TL. Cell-type identification in the autonomic nervous system. Neurosci Bull 2019, 35: 145-155.

17. Mukerjee S, Lazartigues E. Next-Generation tools to study in vivo autonomic regualtion. Neurosci Bull 2019, 35: 113-123. 